\title{
The unusual clinical manifestation of thyroid storm
}

\author{
Claudia Agabiti Rosei - Carlo Cappelli · \\ Massimo Salvetti • Maurizio Castellano • \\ Maria Lorenza Muiesan • Enrico Agabiti Rosei
}

Received: 2 July 2010/ Accepted: 29 October 2010

(C) SIMI 2010

A 67-year-old woman, with type 2 diabetes mellitus, arterial hypertension and a previous myocardial infarction (treated with PTCA and stenting of the anterior interventricular artery) was referred to the Emergency Department (ED) because of severe weakness and abdominal pain. While in the ED, she had a transient loss of consciousness and was admitted to our Intensive care Unit with abdominal pain, hypokalemia $(2.9 \mathrm{mEq} / \mathrm{L})$, increased liver enzymes (AST 134, ALT 303), and a brain CT scan negative for hemorrhagic/or space occupying lesions.

Two weeks prior, because of unstable angina, the patient had undergone a coronary angiography with iodine-containing radiographic contrast agent, showing the lack of restenosis or worsening of coronary atherosclerosis.

At admission, the patient had light dyspnoea, body temperature of $37.8^{\circ} \mathrm{C}$ and sinus tachycardia (110 beats/ min) with mild agitation. Blood pressure was 105/ $74 \mathrm{mmHg}$. The ECG showed ST segment depression. At echocardiography, ejection fraction was $>80 \%$, with left ventricular eccentric hypertrophy (LVMI $57 \mathrm{~g} / \mathrm{m}^{2.7}$ ) and impaired diastolic function. The Holter ECG monitoring showed regular tachycardia with a few short episodes of atrial fibrillation. Laboratory findings revealed thyrotoxicosis $\quad(\mathrm{TSH}<0.004 \mathrm{mUI} / \mathrm{L}, \quad \mathrm{fT} 3>30 \mathrm{pg} / \mathrm{mL}, \quad$ fT4 $>$ $60 \mathrm{pg} / \mathrm{mL}$ ) and the presence of a multi-nodular goiter was shown on a cervical ultrasound study.

The patient was treated with bisoprolol $(10 \mathrm{mg})$ daily, methimazole $30 \mathrm{mg}$ every $6 \mathrm{~h}$ and after 1 week fT 4 and

C. Agabiti Rosei - C. Cappelli - M. Salvetti - M. Castellano ·

M. L. Muiesan $(\varangle) \cdot$ E. Agabiti Rosei

Department of Medical and Surgical Sciences,

Clinica Medica, University of Brescia, c/o 2a Medicina

Generale, Spedali Civili, 25123 Brescia, Italy

e-mail: muiesan@med.unibs.it
fT3 significantly decreased (19.9 and $4.7 \mathrm{pg} / \mathrm{mL}$, respectively). Symptoms improved, and Holtermontor ECG confirmed effective heart rate control.

The patient was discharged with the diagnosis of thyroid storm with newly diagnosed multinodular goiter.

Thyroid storm is a rare life-threatening condition, and is most common in women in their third through sixth decades of life [1], more frequently observed among patients affected by Graves' disease. It is often worsened by precipitating events such as surgery, sepsis, cardiovascular accident or trauma, poor compliance to specific therapy, low socioeconomic status and co-morbid conditions.

Thyroid storm is a manifestation of thyrotoxicosis in which a markedly hypermetabolic state is present. Criteria for a clear distinction between thyrotoxicosis and a thyroid storm are not established, although Burch et al. [1] have suggested a worthwhile clinical predictor score (Table 1). This diagnostic approach allows an easy and prompt recognition of an impending thyroid crisis without any personal bias, committing an early aggressive therapeutic management with full support of the patient in an intensive care unit.

Nevertheless, the diagnosis of thyrotoxicosis still remains inadequately recognized [2,3], and the mortality rate for thyroid storm remains elevated $(>30 \%)$, consequent to serious complications such as acute heart failure.

We report a case of unsuspected hyperthyroidism, presenting a score highly suggestive of thyroid storm (45 points), without any previous thyroid disease.

Abdominal pain is an unusual symptom, although reported in the Bursch score, possibly due to the increased gut motility (with consequent hyperdefecation, malabsorption and weight loss). Vomiting has also been reported, in addition to abnormalities in liver function tests, 
Table 1 Score for the diagnosis of thyroid storm

\begin{tabular}{|c|c|}
\hline Diagnostic parameters & $\begin{array}{l}\text { Scoring } \\
\text { points }\end{array}$ \\
\hline \multicolumn{2}{|l|}{ Thermoregulatory dysfunction } \\
\hline \multicolumn{2}{|l|}{ Temperature ${ }^{\circ} \mathrm{F}\left({ }^{\circ} \mathrm{C}\right)$} \\
\hline $99-99.9(37.2-37.7)$ & 5 \\
\hline $100-100.9(37.8-38.2)$ & 10 \\
\hline $101-101.9(38.3-38.8)$ & 15 \\
\hline $102-102.9(38.9-39.2)$ & 20 \\
\hline $103-103.9(39.3-39.9)$ & 25 \\
\hline$>104.0(>40.0)$ & 30 \\
\hline \multicolumn{2}{|l|}{ Central nervous system effects } \\
\hline Absent & 0 \\
\hline Mild (agitation) & 10 \\
\hline Moderate (delirium, psychosis, extreme lethargy) & 20 \\
\hline Severe (seizures, coma) & 30 \\
\hline \multicolumn{2}{|l|}{ Gastrointestinal-hepatic dysfunction } \\
\hline Absent & 0 \\
\hline Moderate (diarrhea, nausea/vomiting, abdominal pain) & 10 \\
\hline Severe (unexplained jaundice) & 20 \\
\hline \multicolumn{2}{|l|}{ Cardiovascular dysfunction } \\
\hline \multicolumn{2}{|l|}{ Tachycardia (beats/minute) } \\
\hline $90-109$ & 5 \\
\hline $110-119$ & 10 \\
\hline $120-129$ & 15 \\
\hline $130-139$ & 20 \\
\hline$>140$ & 25 \\
\hline \multicolumn{2}{|l|}{ Congestive heart failure } \\
\hline Absent & 0 \\
\hline Mild (pedal edema) & 5 \\
\hline Moderate (bibasilar rates) & 10 \\
\hline Severe (pulmonary edema) & 15 \\
\hline \multicolumn{2}{|l|}{ Atrial fibrillation } \\
\hline Absent & 0 \\
\hline Present & 10 \\
\hline \multicolumn{2}{|l|}{ Precipitating event } \\
\hline Absent & 0 \\
\hline Present & 10 \\
\hline
\end{tabular}

Adapted from [6]

A score of 45 or more is highly suggestive of thyroid storm; a score of 25-44 supports the diagnosis, and a score below 25 makes thyroid storm unlikely

particularly high serum alkaline phosphatase concentrations, and more rarely cholestasis.

Thyroid storm almost always develops in patients with long-standing hyperthyroidism often precipitated by an acute event identified at least "a posteriori" such as surgery, diabetic ketoacidosis, infection, congestive cardiac failure, ingestion of excessive amounts of exogenous iodine and discontinuation of antithyroid drugs. The probability of a hyperthyroidism to deteriorate into a thyroid storm usually represents the consequence of the metabolic demand increase associated with infection, pulmonary embolism or drug reaction; more recently, the use of iodine contrast has frequently become a cause of acute worsening of thyroid hyperfunction.

Heart failure is most commonly seen in the setting of prolonged and severe hyperthyroidism, especially in patients with underlying ischemic or hypertensive heart disease. This condition is well represented by our patient in whom echocardiography confirmed left ventricular hypertrophy and diastolic dysfunction. The patient was complaining of severe weakness, probably due to the relatively rapid development of tachycardia and hypotension.

The appropriate treatment for impending or established thyroid storm is well known, and based on the use of betablockers to antagonize the hyperadrenergic manifestations of severe hyperthyroidism, along with thionamides for the prevention of the new synthesis of thyroid hormone [1]. Another important parameter in the treatment of thyroid storm is represented by the inhibition of the T4 to T3 conversion by blocking the $5^{\prime}$-monodeiodinase enzyme. Steroids and inorganic iodine are both potent inhibitors of this enzyme. In diabetic patients, as in the case we present, the use of corticosteroids can worsen glycemic control, and a strict evaluation of glycemia values, in addition to changes in antidiabetic drugs, may be necessary.

The role of the therapeutic use of iodine-containing agents is still a matter of discussion since the excess or the deficiency of iodine can lead to thyroid dysfunction. Sources of excess iodide include over-the-counter and prescription medications that may be ingested or applied to the skin, dietary supplements and radiographic contrast agents. Some of these substances, such as amiodarone, are stored in fat tissue and may provide excess iodide for months after the last dose is consumed. On the other hand, the use of iodine-containing drugs as a prevention of iodine organification in the thyroid gland is based on a protective mechanism called Wolff-Chaikoff effect [4]. This is an autoregulatory phenomenon, spontaneously reversible, which inhibits formation of thyroid hormones inside the thyroid follicle. It lasts for several days (around 10 days), after which it is followed by an "escape phenomenon" which is typified by resumption of normal organification of iodine and normal thyroid peroxidase function. "Escape phenomenon" occurs because high levels of intracellular iodide suppress the transcription of thyroid peroxidase enzyme, along with NADPH oxidase. The downregulation of transcription of colloid enzymes that attach iodide to thyroglobulin causes a reduction in the synthesis of the downstream product, thyroxin. The Wolff-Chaikoff effect can be used to treat hyperthyroidism by administration of a large amount of iodine. 
The major restraint to the use of inorganic iodine drugs is represented by the possible worsening of hyperthyroidism (providing substrate for new hormone synthesis) and leading to resistance to conventional treatment; treatment with iodine should, therefore, be started only after thionamides have been administered. In addition, in patients with thyroid storm, the therapeutic efficacy of inorganic iodine is greater when the serum iodine pool is not increased. In the case we present, inorganic iodine was not administered for the treatment of thyroid storm, because the serum iodine pool was increased after the coronary angiography with iodine contrast was performed.

At the present time, little is known about the use of iodine-containing contrast media with conflicting data in the literature [5]. A low risk of iodine-induced thyrotoxicosis was reported in euthyroid patients, but scarce information is available about the risk in patients with pre-existing partial or total suppression of the TSH secretion. Few available information suggest that only patients with known thyroid gland functional abnormalities should be considered to be at high risk for worsening of a preexisting thyroid dysfunction after administration of iodine containing contrast.
We suggest the level of thyroid stimulating hormone as part of the initial emergency laboratory assessment in patients who undergo diagnostic tests with iodine-containing contrast.

Conflicts of interest None.

\section{References}

1. Sarlis N, Gourgiotis L (2003) Thyroid emergencies. Rev Endocr Metab Disord 4:129-136

2. Ngo SY, Chew HC (2007) When the storm passes unnoticed-a case series of thyroid storm. Resuscitation 73:485-490

3. Stanzani Maserati M, Faustini Fustini M (2009) Thyroid storm with atypical neurological signs: an unusual clinical emergence of a life-threatening event. Intern Emerg Med 4:181-182

4. Eng PH, Cardona GR, Fang SL et al (1999) Escape from the acute Wolff-Chaikoff effects is associated with decrease in thyroid sodium/iodine symporter messenger RNA and protein. Endocrinology 140:3404-3410

5. van der Molen A, Thomsen S, Morcos S (2004) Effect of iodinated contrast media on thyroid function in adults. Eur Radiol 14:902-907

6. Burch HB, Wartofsky L (1993) Life-threatening thyrotoxicosis. Thyroid storm. Endocrinol Metab Clin North Am 22:263 